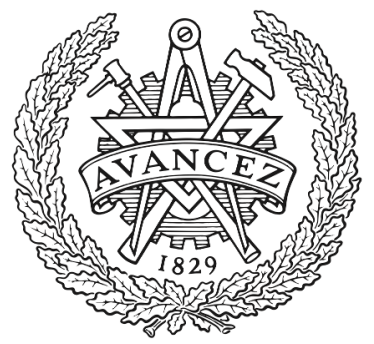

CHALMERS

UNIVERSITY OF TECHNOLOGY

\title{
Longitudinal velocity and road slope estimation in hybrid electric vehicles employing early detection of excessive wheel slip
}

Downloaded from: https://research.chalmers.se, 2023-04-26 10:39 UTC

Citation for the original published paper (version of record):

Klomp, M., Gao, Y., Bruzelius, F. (2014). Longitudinal velocity and road slope estimation in hybrid electric vehicles employing early detection of excessive wheel slip. Vehicle System Dynamics, 52(SUPPL. 1): 172-188.

http://dx.doi.org/10.1080/00423114.2014.887737

N.B. When citing this work, cite the original published paper. 


\title{
Longitudinal Velocity and Road Slope Estimation in Hybrid Electric Vehicles Employing Early Detection of Excessive Wheel Slip Matthijs Klomp ${ }^{1}$, Gao Yunlong ${ }^{2}$, Fredrik Bruzelius ${ }^{3}$ \\ ${ }^{1}$ Vehicle Dynamics \& Controls, e-AAM Driveline Systems, Trollhättan, Sweden \\ ${ }^{2}$ Clean Energy Automotive Engineering Center, Tongji University, Shanghai, China \\ ${ }^{3}$ Department of Applied Mechanics, Chalmers University of Technology, Gothenburg Sweden e-mail of lead author: matthijs.klomp@aam.com
}

\begin{abstract}
Vehicle speed is one of the important quantities in vehicle dynamics control. Estimation of the slope angle is in turn a necessity for correct dead reckoning from vehicle acceleration. In the present work, estimation of vehicle speed is applied to a hybrid vehicle with an electric motor on the rear axle and a combustion engine on the front axle. The wheel torque information, provided by electric motor, is used to early detect excessive wheel slip and improve the accuracy of the estimate. A best-wheel selection approach is applied as the observation variable of a Kalman Filter which reduces the influence of slipping wheels as well as reducing the computational effort. The performance of the proposed algorithm is illustrated on a test data recorded at a winter test ground with excellent results, even for extreme conditions such as when all four wheels are spinning.
\end{abstract}

Keywords: velocity estimation, Kalman filter, wheel torque, best wheel speed, slope estimation

\section{INTRODUCTION}

Accurate knowledge of the vehicles longitudinal velocity is essential for example wheel slip control and the slope angle is important for real-life fuel economy optimization and improved traction control. The task of velocity estimation is challenging when driving all four wheels and particularly in slippery conditions.

Direct measurement of the longitudinal velocity, as opposed to estimation, is too expensive and/or impractical for vehicle applications. Therefore, the longitudinal velocity needs to be estimated from the wheel speed sensors, accelerometers, wheel torque information as well as other sensors.

These aforementioned sensor sources each have limitations; the wheel speeds become unrelated to the vehicle velocity during excessive wheel slip and time integration of the longitudinal acceleration accumulates sensor bias causing the estimation to drift. Another source of error is the gravitational component acting in the direction of the road slope contaminating the acceleration measurement. Hence, estimation of the road slope angle is necessary.

In order to circumvent the shortcomings of each individual measurement method, it is necessary to employ sensor fusion. Vehicles with electric drive system have more accurate information of the wheel torque compared to conventionally propelled vehicles. This paper explores how this improved accuracy can be used to improve the estimation of the longitudinal velocity.

As mentioned, vehicle speed is one of the important quantities in vehicle dynamics control and estimation of it has consequently drawn substantial attention in both academic research as well as in the industry. This fact is confirmed by the amount of published work in the field. Previously reported approaches ranges from relaying on auxiliary sensors such as microphones in [8] to optical sensors as in [9]. However, the most common approach in the literature is based on estimation using primarily the wheel speed sensors. It is typical to combine these with accelerometers and inertial measurement unit systems in general and other available sensors in modern vehicles. Simple heuristic approaches of selecting the best candidate, i.e. with the smallest wheel slip can be found in for example [10, 11]. These heuristic methods have obvious advantages and are often popular in automotive industry due to their simplicity and are easy to implement. Improvement of the performance by introducing weighted dead reckoning from accelerometers can be found in [2, 3, 4]. Incorporation of models and additional sensor information can be found in for example [12] and [13]. This further improves the performance of the estimator, but to the cost of structural complexity, computational burden as well as robustness to model or sensor errors.

In this work a similar approach as in [1] is taken, namely using simple kinematic models in combinations with wheel speed sensors and conventional linear Kalman filers in ordinary working conditions and using dead reckoning of accelerometers for extreme conditions such as four excessively spinning wheels. The present work extends the work in [1] with an enhanced algorithm to more rapidly detecting excessive wheel slip by utilizing the force information from the hybrid electric propulsion system. This rapid detection of excessive wheel slip is essential to minimize the initial estimation offset when starting the dead reckoning. A strategy of using only one best wheel strategy in the Kalman filer further improves robustness of the estimation.

This paper is organized as follows. The algorithm is developed in chapter 2, and chapter 3 introduces the test vehicle. Then the test results analysis are explained in chapter 4. Finally, the conclusions are drawn in chapter 5. 


\section{ALGORITHM DESIGN}

The estimator used in this paper is based on the standard linear quadratic estimation filter, known as the Kalman filter. The prediction of the velocity is made as by integration of the slope compensated longitudinal acceleration and the measurement update is made using the best-wheel measurement.

As mentioned in the introduction, the accuracy of this Kalman filter could be improved in two aspects. One is to find excessive wheel slip in time, the other is the best-wheel speed selection. Hence, the algorithm in this paper is designed based on these principles. As shown in Figure 1, this algorithm is composed of five parts. Apart from the Kalman filter for velocity estimation, the other parts, such as wheel velocity transformation, slope estimation (an additional Kalman filter), wheel speed selection and excessive slip criteria are other key components in the overall algorithm.

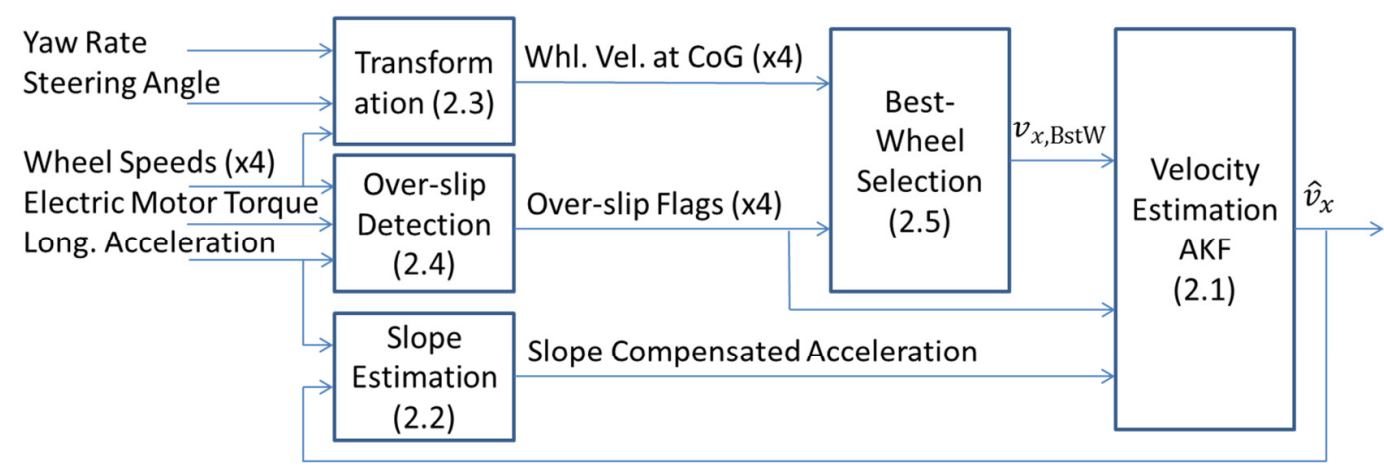

Figure 1 Algorithm overview with corresponding section numbers in parenthesis

The general discrete system Kalman filter function consists of two parts; a prediction step using knowledge about the system behavior and the inputs to the system and a measurement update, fusing the prediction with measurements:

$$
\begin{gathered}
\hat{x}_{k \mid k-1}=\Phi_{k-1} \hat{x}_{k-1 \mid k-1}+\Psi_{k-1} u_{k} \\
P_{k \mid k-1}=\Phi_{k \mid k-1} P_{k-1 \mid k-1} \Phi_{k \mid k-1}^{\mathrm{T}}+Q_{k \mid k-1} \\
K_{k}=P_{k \mid k-1} H_{k}^{\mathrm{T}}\left(H_{k} P_{k \mid k-1} H_{k}^{\mathrm{T}}+R_{k \mid k-1}\right)^{-1} \\
\hat{x}_{k \mid k}=\hat{x}_{k \mid k-1}+K_{k}\left(z_{k}-H_{k} \hat{x}_{k \mid k-1}\right) \\
P_{k \mid k}=\left(I-K_{k} H_{k}\right) P_{k \mid k-1}
\end{gathered}
$$

where $\hat{x}_{k \mid k}$ is the post measurement update estimation states vector at the discrete time step $k, \hat{x}_{k \mid k-1}$ is the preestimation (prediction) of the states at step $k$ and $u$ is the inputs vector and $z$ is the observation variables (measurements) vector. Moreover, $P, Q$ and $R$ are, respectively, the estimation error, system error and measurement error covariance matrices. Finally, $H_{k}$ is the observation matrix, $K_{k}$ is the Kalman filter gain matrix and $\Phi$ and $\Psi$ are, respectively, the system matrix and input matrices.

\subsection{Velocity Estimation}

For the velocity estimation Kalman filter (index $v$ ) we choose

$$
\hat{x}_{v}=\hat{v}_{x}, u_{v}=\dot{v}_{x}, z_{v}=v_{x, \mathrm{BstW}}
$$

where $\hat{v}_{x}$ is the estimated longitudinal velocity, $\dot{v}_{x}$ is the slope-compensated longitudinal acceleration (section 2.2) and $v_{x, \text { BstW }}$ is the measured velocity based on the selected best-wheel speed (section 2.5). Further, we identify that

$$
\Phi_{v}=1, \Psi_{v}=\tau, H_{v}=1
$$

where $\tau$ is the sample time of the filter. Further, we choose in this work 


$$
Q_{v}=1, R_{v}=200
$$

to estimate the velocity. These gains can are tuning variables and need to be calibrated depending on the application. Note that this Kalman filter is scalar, thus greatly simplifying the necessary computations compared to use all four wheels.

A key point in the present work is that the Kalman filter gain matrix $K$ is controlled directly based on rapid detection of excessive slip. When excessive slip of the best wheel is detected (section 2.4), the control gain matrix $K$ and set it to zero implying dead reckoning of the longitudinal acceleration. In case of no excessive slip of the best wheel, $K$ is updated as in a typical Kalman filter.

\subsection{Slope Estimation}

In general, the measurement of accelerometer is composed of both the acceleration of movement and the gravity acceleration along the road slope as well as the lateral velocity component.

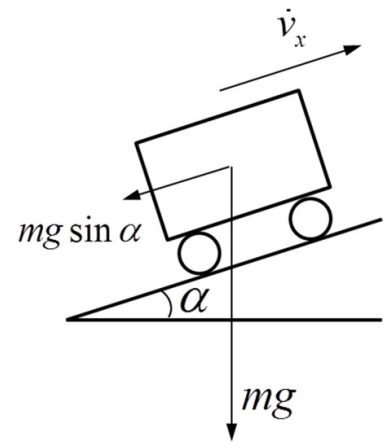

Figure 2 Vehicle on sloped road

As partly shown in Figure 2, the measurement of accelerometer can be written as

$$
a_{x}=\dot{v}_{x}+v_{y} \dot{\psi}+g \sin \alpha
$$

where $a_{x}$ is accelerometer measurement, $\dot{v}_{x}$ is slope compensated longitudinal acceleration, $g$ is gravity acceleration and $\alpha$ is road slope angle. Further, $v_{y} \dot{\psi}$ is the product of the lateral velocity, $v_{y}$, and the yaw rate, $\dot{\psi}$. In this work, the lateral velocity is assumed to be negligible.

Choosing $v_{x}$ and $\sin \alpha$ as state variables, $a_{x}$ as the input variable and $\hat{v}_{x}$ as observation variable we have that

$$
x=\left[\begin{array}{ll}
v_{x} & \sin \alpha
\end{array}\right]^{\mathrm{T}}, u=a_{x}, z=\hat{v}_{x}
$$

where $v_{x}$ and $\hat{v}_{x}$ are the vehicle velocity and its estimation result, respectively.

The state equation can then be written as

$$
\dot{x}=\left[\begin{array}{cc}
0 & -g \\
0 & 0
\end{array}\right] x+\left[\begin{array}{l}
1 \\
0
\end{array}\right] u
$$

Then, discretize the state function and substitute

$$
\Phi_{\alpha}=\left[\begin{array}{cc}
1 & -\tau g \\
0 & 1
\end{array}\right], \Psi_{\alpha}=\left[\begin{array}{l}
1 \\
0
\end{array}\right], H_{\alpha}=\left[\begin{array}{ll}
1 & 0
\end{array}\right]
$$

into equation (1) to estimate the road slope. The prediction and measurement covariance matrices are chosen as:

$$
Q_{\alpha}=\left[\begin{array}{cc}
10 & 0 \\
0 & 0.1
\end{array}\right], R_{\alpha}=200
$$

The observation variable of slope estimation algorithm is the output of velocity estimation, and the result of slope estimation is used to fix the accelerometer measurement. Then these two algorithms are combined together. 
However, when all of there is excessive wheel slip simultaneously on all four wheels on a sloped road, neither the wheel speeds nor the acceleration is reliable in this context. The velocity and slope estimation will be ruined at that time.

In order to solve this problem for real driving scenarios, it supposed that the road slope does not change rapidly. Thus, we set the gradient limit on the slope estimation. If the slope estimation does not meet with this criterion,

$$
|\alpha(k)-\alpha(k-1)|<\Delta \alpha
$$

then the slope estimation result maintains the value obtained from previous time-step.

\subsection{Wheel Speed Transformation to Center of Gravity}

Before the four wheel contact patch velocities, based on wheel rotational speed measurement and tire radius estimation (not considered here), can be compared, each wheel velocity needs to be transposed to the center of gravity. Only after that the wheel speeds can be used to find the observation variable. Take front left wheel for example, by linear transformation we find that the longitudinal velocity in the wheel coordinates is

$$
v_{f l}=\left(v_{x}-s_{f} \dot{\psi}\right) \cos \delta+\left(v_{y}+l_{f} \dot{\psi}\right) \sin \delta
$$

where, $s_{f}$ is half the front track width, $l_{f}$ is the distance between front axle and gravity center and $\delta$ is the steered angle of the wheel. Since, by definition,

$$
v_{y}=v_{x} \cos \beta
$$

where $\beta$ is vehicle side slip angle we can, by combining (11) and (12), find that

$$
v_{x}=\frac{v_{f l}+\left(s_{f} \cos \delta-l_{f} \sin \delta\right) \dot{\psi}}{\cos \delta+\tan \beta \sin \delta}=v_{f l} \sec \delta+\left(s_{f}-l_{f} \tan \delta\right) \dot{\psi}
$$

for $\beta=0$. Similarly the relationship between the other wheel speeds and the vehicle velocity can be derived.

\subsection{Best-wheel Selection}

As described in above, this algorithm only uses one wheel-speed-based velocity measurement as the input of Kalman filter in each sample time. Therefore, the next step is to find which of these four wheel-speed measurements that best represent the vehicle velocity; the so-called best wheel. This wheel should be the wheel with the smallest wheel slip. The strategy to find the best wheel is select from the wheels without excessive slip by choosing the minimum wheel speed in traction and the maximum speed when braking. When all four wheels show excessive slip one chooses similarly among all of the wheels. This strategy is summarized in Table 1. The excessive slip flag of each wheel will also accompany the chosen speed. How to find this excessive slip flag is described in the next section.

Table 1 Best wheel selection strategy

\begin{tabular}{cll}
\hline Excessive slip count & \multicolumn{1}{c}{ Traction } & \multicolumn{1}{c}{ Braking } \\
\hline 0 & Minimum of 4 wheel speeds & Maximum of 4 wheel speeds \\
$1 \sim 3$ & Minimum of reliable wheel speeds & Maximum of reliable wheel speeds \\
4 & Minimum of 4 wheel speeds & Maximum of 4 wheel speeds \\
\hline
\end{tabular}




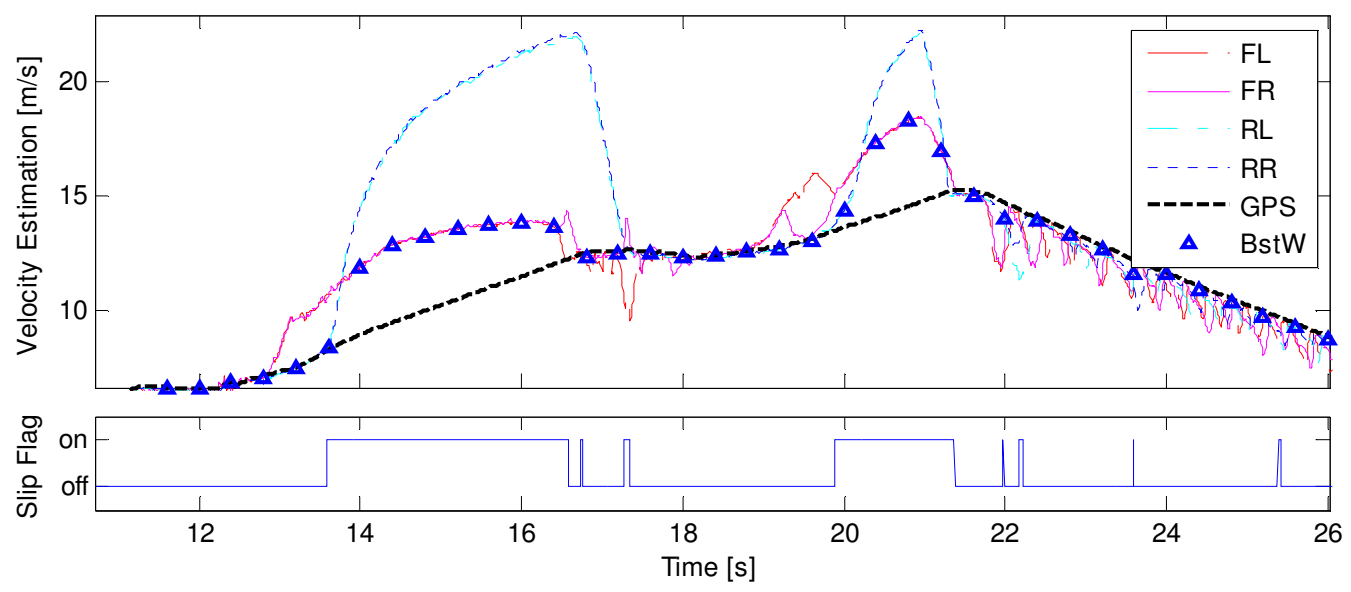

Figure 3 Wheel speed selection

\subsection{Excessive Slip Detection}

Selecting which wheel that is excessive slip early and in a robust way, is the core of the algorithm presented in this paper.

There will be three excessive slip criteria introduced in this section. These are the wheel speed criterion, preestimation criterion and wheel torque criteria. If any of these criteria is satisfied, the wheel is decided to be excessive slip.

\subsubsection{Wheel Speed Change Criterion}

The aim of wheel speed change criterion is to detect rapid changes in the wheel speed, $\omega$, relative to an average over $m$ time samples, as an indication of over slip. The criterion is defined as true when

$$
\left|\omega(k)-\frac{1}{m} \sum_{i=k-m}^{k-1} \omega(i)\right|>\Delta \omega_{\text {thrshld }}
$$

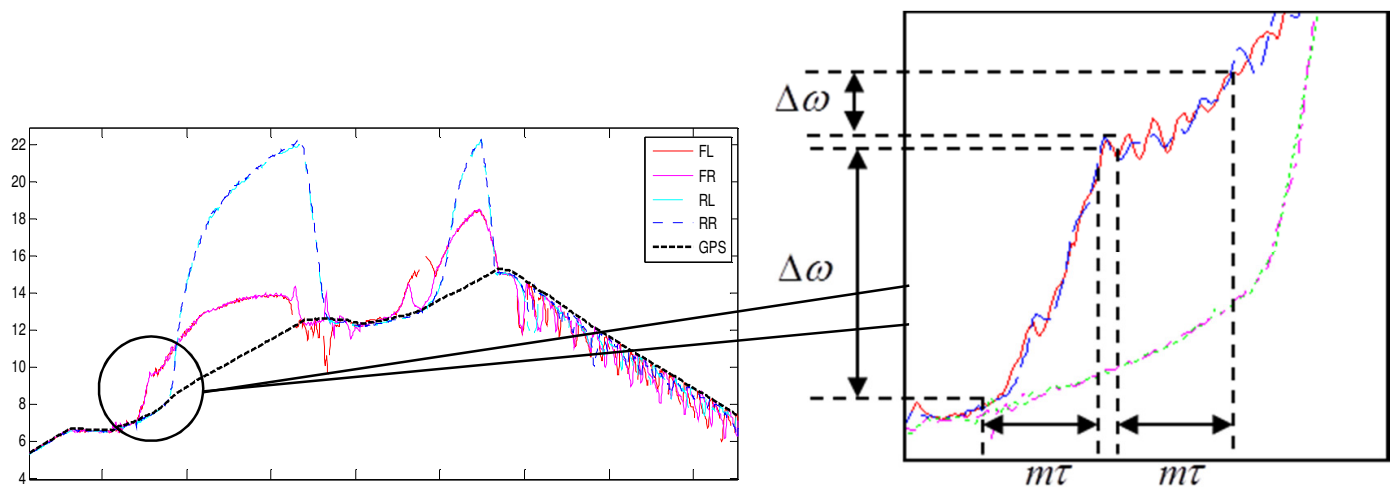

Figure 4 Wheel speed criteria

As shown in Figure 4, when the speed measurement changes rapidly, it assumed to be excessive slip. However, after the beginning of excessive slip, the measurement turns out to be flat. Thus, the threshold should be a small value to detect all sections of excessive slip. The problem is that the threshold cannot be too small; otherwise it will be ruined by the measurement noise. On the other hand, if the threshold is too large, it will cause obvious delay or miss detection of excessive slip. Hence, there is a compromise between the delay in slip detection and sensitivity to noise in this criterion. 


\subsubsection{Pre-estimation Deviation Criterion}

The second excessive slip criterion is based on the difference between the wheel speeds multiplied by the tire radius, $R_{r}$, and the pre-estimation of the velocity, $\hat{v}_{x}^{-}$. The criterion is written as

$$
\left|\omega(k) R_{r}-\hat{v}_{x}^{-}(k)\right|>\Delta v_{\text {thrshld }}
$$

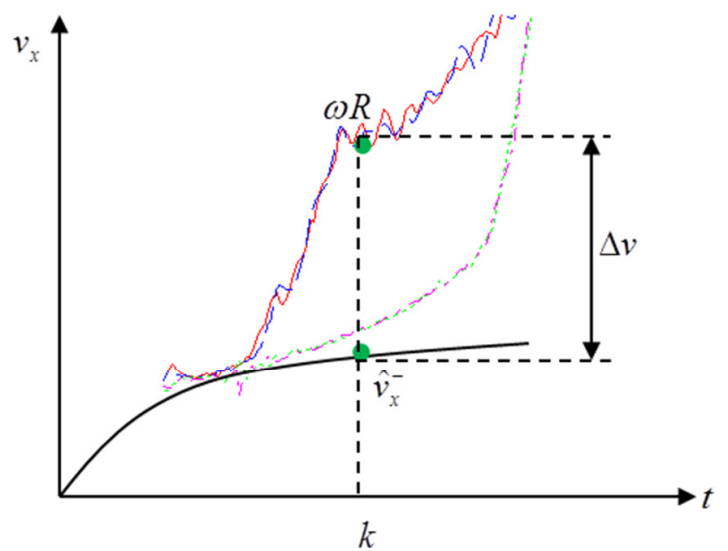

Figure 5 Pre-estimation criteria

To solve the problem with the noise sensitivity associated with the wheel speed change criterion, the pre-estimation velocity is used to find out the slipping wheels. As shown in Figure 5, even if the wheel speed turns flat, there is still a significant deviation from the pre-estimation value. Thus, according to this criterion, we can find the excessive slip even though the measurement does not change intensely. Also, the threshold here cannot be too small.

The proper threshold of the criteria can distinguish excessive slip and measurement noise. Also, it causes the delay of slipping detection, and then affects the accuracy of the velocity estimation. As shown in Figure 6 (left), when all the four wheels are over slip, the velocity estimation will be replaced by the integration of the acceleration. However, because of the delay of excessive slip detection, the initial value is not correct and the estimation error is obvious.

On the other hand, the threshold cannot be too strict; otherwise the over slip criteria will be affected by measurement noise. In order to overcome this delay, a third criterion based on wheel torque is used.
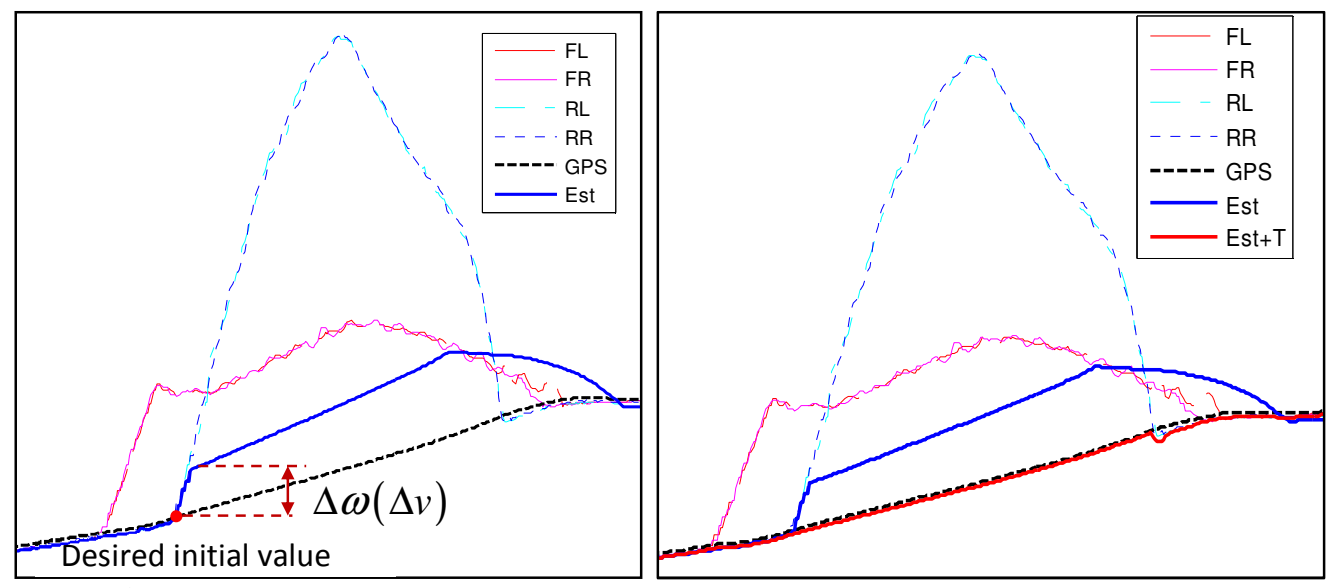

Figure 6 Influence of the excessive slip-detection delay (left) and Improvement of estimation results (right)

\subsubsection{Wheel Torque Criterion}

Generally, the wheel torque can be obtained much more accurately from an electric motor than an internal combustion engine. Hence, we take advantage of the electric motor torque to find out the excessive slip of the wheels. When the axle torque from the motor is greater than the maximum torque that offered by the road friction, the wheel will excessive slip. Take rear axle as example, the normal force on the axle is 


$$
F_{z r}=\frac{m\left(l_{f} g+h a_{x}\right)}{l_{f}+l_{r}}
$$

where $F_{z r}$ is the normal force on the rear axle, $m$ is the total mass of the vehicle, $a_{x}$ is the acceleration from the vehicle center of gravity (CG), $l_{f}$ and $l_{r}$ are, respectively, the distance between CG and front and rear axle, $h$ is the height of the CG (to the earth) and $g$ is the gravity acceleration.

At the time that all wheels are spinning, the maximum of road friction can be assumed to be

$$
\mu_{\max }=\frac{\dot{v}_{x}}{g}
$$

Then, the maximum axle torque is

$$
T_{r \text { max }}=\mu_{\text {max }} F_{z r} R_{r}
$$

where $R_{r}$ is the wheel radius. When the measured rear axle torque $T_{r}$ is greater than $T_{r \max }$ the wheel passes the peak friction and the wheel begins to excessive slip. With this criterion, the estimation result is better than before, as shown in Figure 6 (right), the "Est" curve only uses the first two criteria above and the "Est+T" curve uses all the three criteria.

This criterion, however, is sensitive to measurement noise because we want to find out the excessive slip at the very beginning of slipping. Therefore, change in the wheel speed is taken into consideration to overcome this sensitivity. When the torque measurement meets the slip criterion, if the wheel speed increases rapidly, this means the excessive slip judgment is verified and the wheel is decided to be excessive slip; otherwise, not excessive slip. That is, in a short time period after the detection of excessive torque, the increment of the wheel speed should be greater than the threshold value, see equation (15), and then decide the wheel is slipping. This rapid increase is detected by checking if

$$
\omega(k)-\omega(k-m)>\Delta \omega_{1}
$$

where $\omega(k)$ and $\omega(k-m)$ are respectively the wheel speed measurements at discrete time-step $k$ and $m$ previous time-steps and $\Delta \omega_{1}$ is the excessive slip start threshold value. The excessive slip flag will be latched on after criteria (17) and (18) are both fulfilled. When recovering from excessive slip, the wheel speed should decrease rapidly, see Figure 6. After that, the excessive slip ends and the excessive slip flag is reset by checking if

$$
\omega(k)-\omega(k-m)<\Delta \omega_{2}
$$

where $\Delta \omega_{2}$ is the excessive slip reset threshold value.

To explain the torque criterion graphically, an example is shown in Figure 7. 


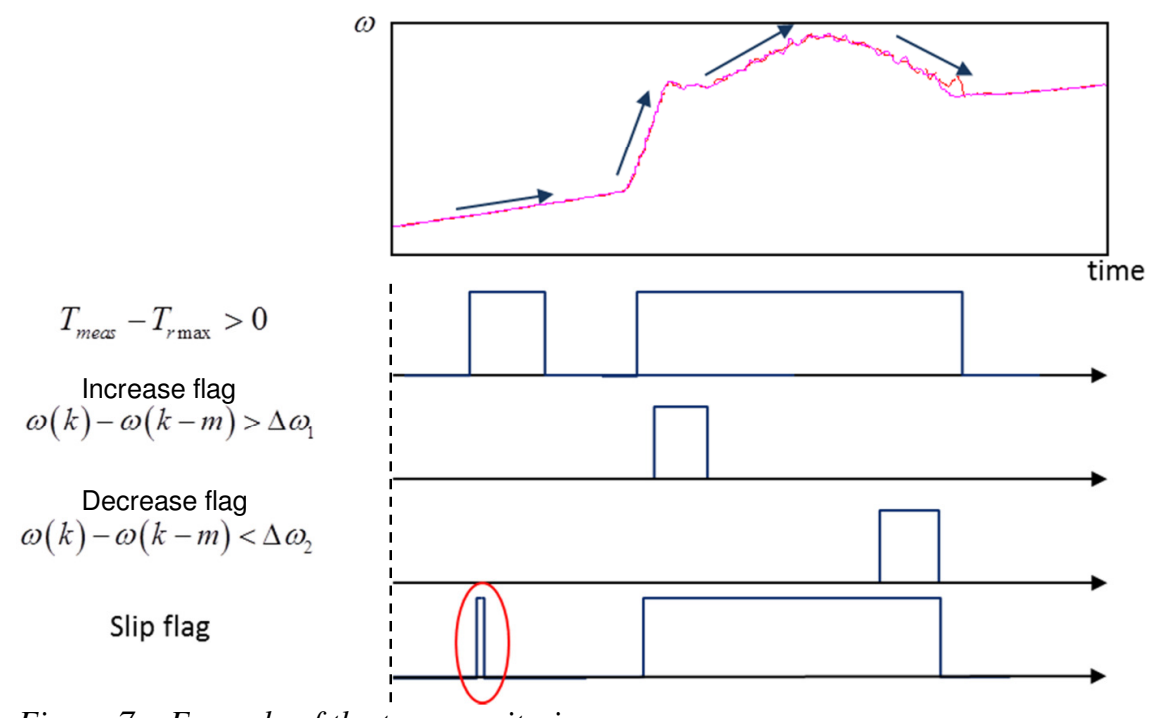

Figure 7 Example of the torque criteria

The first graph in Figure 7 shows the wheel speed measurements. Then, the second one shows the wheel torque flag. If torque measurement is greater than the maximum torque $\left(T_{\max }\right)$, the flag will be activated. The following graphs are "increase flag" and "decrease flag", which are, respectively, activated according to equation (18) and (19). At last, "slip flag" is shown in the fifth graph.

When the torque measurement is greater than maximum torque, but the wheel speed do not change intensely, the slip flag will rise but fall immediately. If the wheel speed measurement increases abnormally after obtaining excessive torque, the "increase flag" is activated. Then, the algorithm is triggered to detect the decrease of the wheel speed. Also, the abnormal decrease will activate the "decrease flag". Finally, we decide the wheel is over slipping from the rising edge of "increase flag" to the falling edge of "decrease flag".

Obviously, there is a pulse in the slip flag (shown in the red circle), which caused by the torque measurement error. But it will not cause huge velocity estimation error. On the other hand, if the torque measurement does not meet with the criterion, we will not check the wheel speed but decide against excessive slip directly.

\section{TEST VEHICLE WITH AN ELECTRIC ALL-WHEEL DRIVE SYSTEM}

The test vehicle, shown right in Figure 8, is a modified passenger vehicle with an internal combustion engine (ICE) driving the front axle and a single electrical motor [7] (rear drive-unit, RDU, see Figure 9) driving the rear wheels. Both front and rear axles have standard open differentials and equal length drive shafts. Since torque estimation is available for both the ICE and the RDU, these can be used as additional inputs to the estimator as well as the electrical motor speed (signed). Verification of the estimation algorithm performance is made using a high-end inertial/GPS platform (IMU) as reference. The parameters of vehicle and motor can be seen in Table 2.
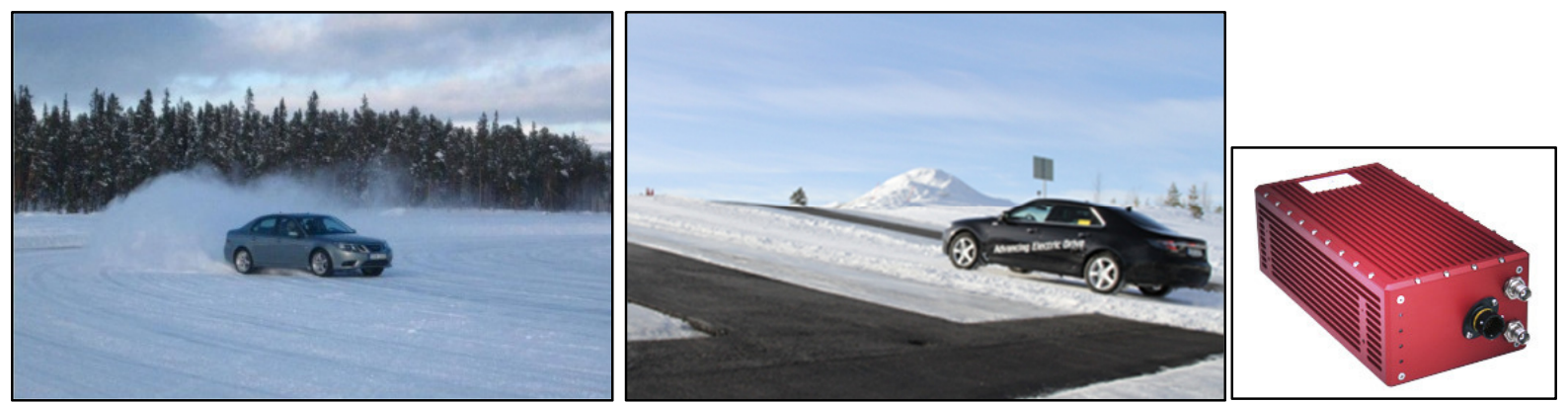

Figure 8 Test vehicles at high side-slip (left) and start at slopes (middle) maneuvers as well as IMU (right) 
Table 2 Parameters of vehicle and powertrain

\begin{tabular}{llll}
\hline Parameter & Value & Parameters & Value \\
\hline Wheel Radius $\left(R_{r}\right)$ & $328.5[\mathrm{~mm}]$ & Peak power (motor) & $50[\mathrm{~kW}]$ \\
Steer Ratio & 15.7 & Peak torque (motor) & $106[\mathrm{Nm}]$ \\
Front Axle to CG $\left(l_{f}\right)$ & $1362[\mathrm{~mm}]$ & Max speed & $15000[\mathrm{rpm}]$ \\
Rear Axle CG $\left(l_{r}\right)$ & $1475[\mathrm{~mm}]$ & Gear ratio (motor) & 11.3 \\
Total Mass of the Vehicle & $1987[\mathrm{~kg}]$ & Peak power (engine) & $110[\mathrm{~kW}]$ \\
Vehicle Track (Front/Rear) & $1583 / 1585[\mathrm{~mm}]$ & Peak torque (engine) & $240[\mathrm{Nm}]$ \\
\hline
\end{tabular}

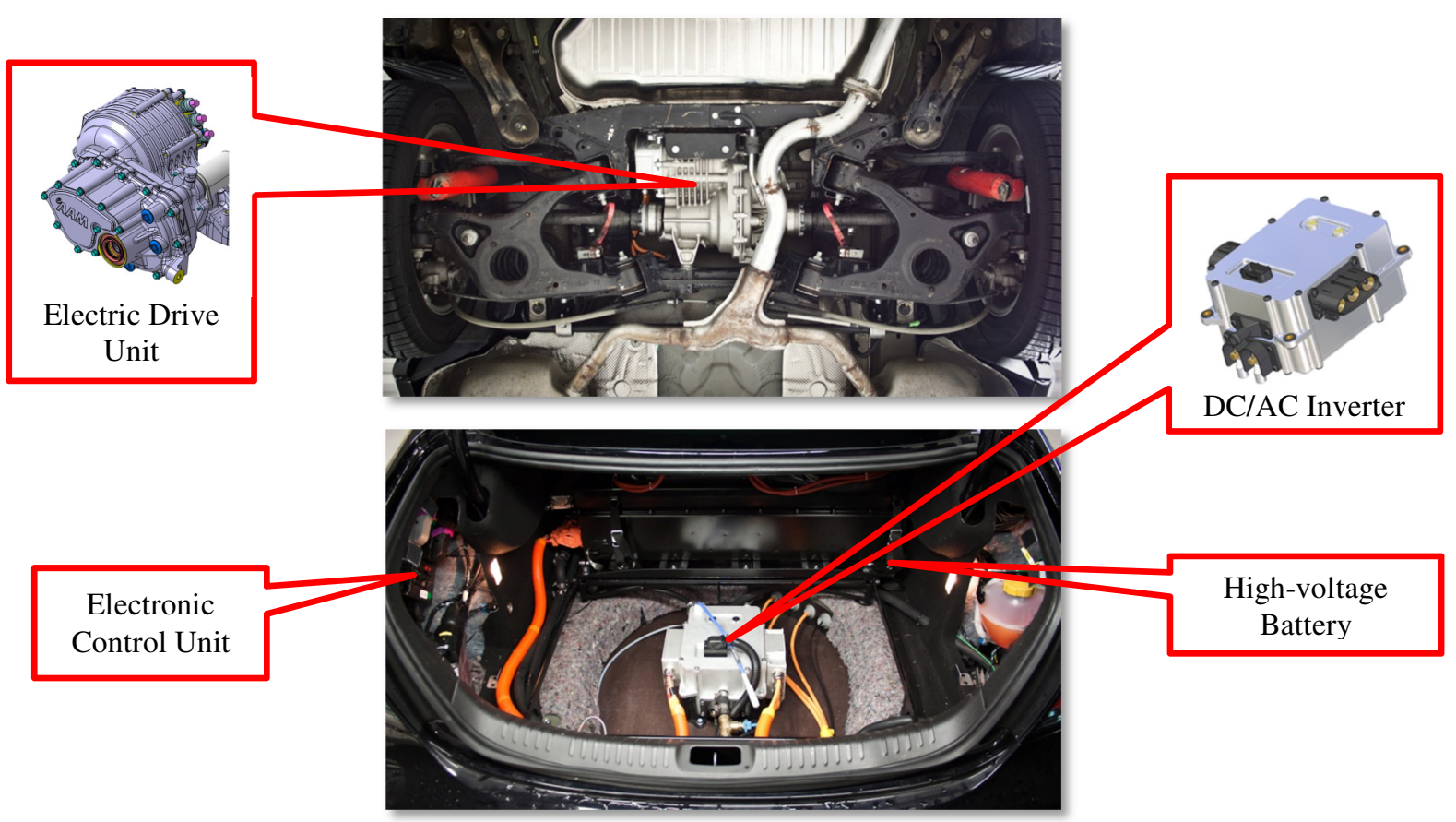

Figure 9 Hybrid-electric Drive System

\section{ON-ROAD TEST IN WINTER CONDITIONS}

The estimation algorithm was evaluated using test measurements which were performed on asphalt, snow, ice on slopes up to $20 \%$ in winter conditions of at a commercial test site in Arjeplog in northern Sweden [6]. As described above, we aim to reduce the delay to detect excessive slip by adding a novel detection method. The purpose of the measurements is to verify the performance of the proposed velocity estimation algorithm. Before the test, we carried out the parameter calibrations first. The parameters which are calibrated off-line are the wheel radius and accelerometer offset. It is possible to perform estimation of also these parameters on-line, but they need longer measurements than those performed here to give satisfactory results. Therefore it was chosen to calibrate these slowly varying parameters off-line.

\subsection{Parameter calibrations}

The parameter calibration work mainly focuses on wheel radius calibration and sensor off-set.

The on-line radius calibration is confronted with problems on low friction road, since the wheel is rather easy to be slipping. Thus, the off-line calibration is carried out before test. During calibration work, the vehicle is driven to $50 \mathrm{~km} / \mathrm{h}$, then coast down. The measurements of wheel speed and reference velocity are recorded in this time period. 
(a)

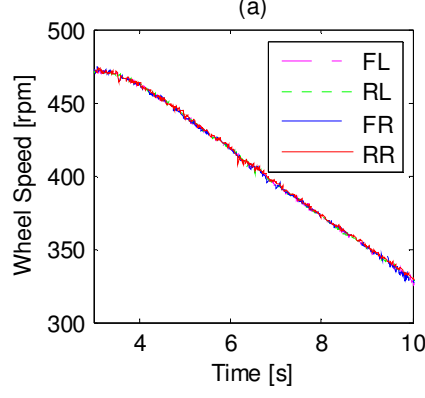

(b)

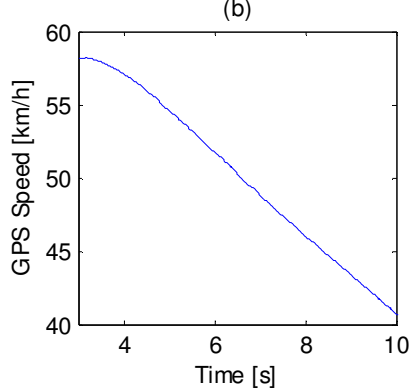

Figure 10 Measurements of wheel speeds (a) and GPS speed (b) during coast-down

Figure 10 is one of several groups of measurements and we use constrained linear regression to find the relation between wheel speeds and the vehicle velocity. This relation is assumed to be

$$
v_{\mathrm{GPS}}(t)=R_{\mathrm{cal}} \widetilde{\omega}(\mathrm{t})
$$

i.e. the offset is assumed to be zero. In (20), $v_{\mathrm{GPS}}$ is the longitudinal velocity measurement of the IMU, $\widetilde{\omega}$ is the mean value of the four wheel speed measurements and $R_{\text {cal }}$ is the calibrated wheel radius. The result of this linear regression gives that the wheel radius is $0.3286 \mathrm{~m}$ and based on all groups of measurement the radius if found to be $0.3285 \mathrm{~m}$.

As for the sensor off-set, one of the most important sensors is longitudinal accelerometer. To find this off-set we set the test vehicle standstill on a flat road, and record the acceleration measurement during a period of time. Then, the accelerometer off-set is the mean value of the measurements. In this work, we take use of road slope estimation algorithm (section 2.2). The state variable and input variable are the same, but here we choose the longitudinal velocity measurement as observation variable.

The measurements on flat road are chosen to calibrate the accelerometer, and then calculate the mean of the slope estimation results. The average slope estimation results on flat road are shown in Table 3.

Table 3 Accelerometer bias from slope estimation results

\begin{tabular}{lccc}
\hline Driving Scenario & bias value $1\left[\mathrm{~m} / \mathrm{s}^{2}\right]$ & bias value $2\left[\mathrm{~m} / \mathrm{s}^{2}\right]$ & bias value $3\left[\mathrm{~m} / \mathrm{s}^{2}\right]$ \\
\hline Acceleration and deceleration on Ice & -0.1498 & -0.1554 & -0.1465 \\
Acceleration and deceleration on Snow & -0.1787 & -0.1676 & -0.1660 \\
Big Circle & -0.1502 & -0.1506 & -0.1506 \\
Charge & -0.1255 & -0.1446 & -0.1198 \\
Circle On Snow & -0.1675 & -0.1691 & -0.1667 \\
Circle On Ice & -0.1729 & -0.1662 & -0.1227 \\
Coast Down & -0.1347 & -0.1222 & -0.1314 \\
Driving On Ice & -0.1008 & -0.1157 & -0.1089 \\
Parking & -0.1650 & -0.1635 & -0.1469 \\
Start On Ice & -0.1520 & -0.1665 & -0.1531 \\
Slalom & -0.1753 & -0.1673 & -0.1701 \\
\hline
\end{tabular}

Averaging the measurements shown in Table 3, the accelerometer bias is concluded to be

$$
a_{x, \mathrm{offset}} \approx-0.15\left[\mathrm{~m} / \mathrm{s}^{2}\right]
$$

\subsection{Test results}

As shown in Figure 11, the vehicle starts on polished ice from stand still. In this and subsequent figures, eight different speeds are shown. These are the four wheel speeds transformed into equivalent vehicle velocities, the "best wheel" speed, the reference velocity from the GPS system and two estimations from the previous (without torque information) and present work. Further, the estimation error, slip flag and slope estimation are shown. The aim of the estimation is to stay within $\pm 5 \%$ of the true vehicle speed. This error target is somewhat arbitrary, since what is most important for the slip control is firstly to have the correct sign of the wheel slip (normalized relative wheel 
speeds) and secondly to have the correct absolute value of the wheel slip. As can be seen in In Figure 11 and Figure 12, the estimation from Gao2012 [1] is above the wheel speeds around time 9.5-10.5 [s] and 15-16 [s], respectively, which would result in the erroneous conclusion that the wheels have a large brake slip.

In Figure 11 the slip flag turns to "on" as soon as all the wheels excessive slip. The current estimation result (red curve) follows the reference velocity closely, even if all the wheels are slipping simultaneously. By the help of excessive slip criteria, the algorithm starts to calculate the integration of acceleration without delay and the results are better than the method without motor torque (blue dash-dotted curve). It can also be seen in the error plot, the error of current method is very small.

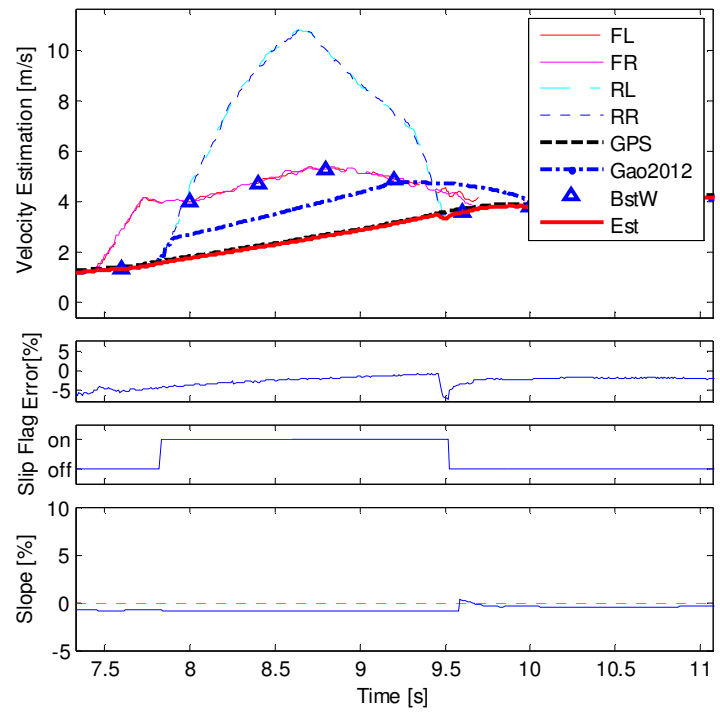

Figure 11 Test results of start on polished ice
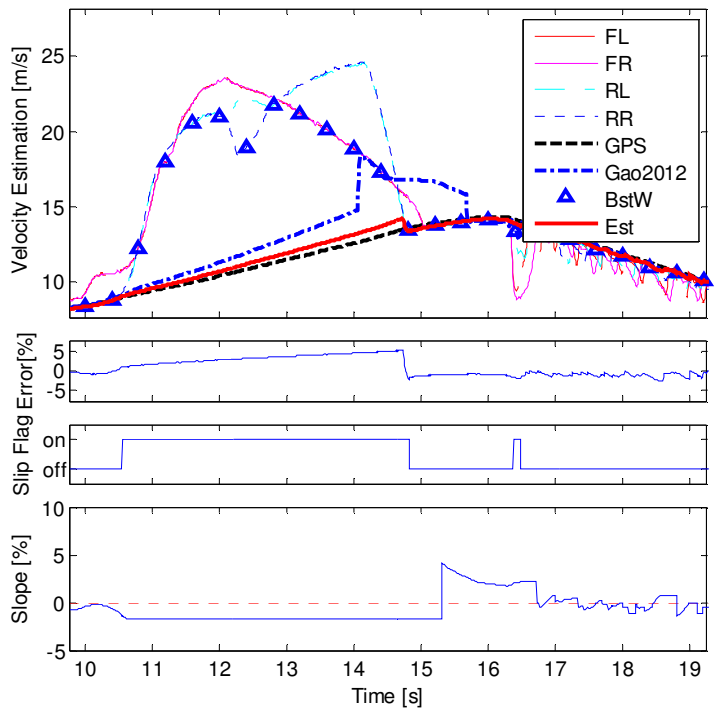

Figure 12 Test results of acceleration on polished ice

In Figure 12 results from are shown when accelerating on polished ice. All four wheels over slip, but the velocity estimation results are accurate during this challenging situation. There is some over-estimation in velocity because of the bias of slope estimation but the estimation error remains within $\pm 5 \%$.

Next, the vehicle is driven on circle road covered by ice and the vehicle speed is at $50 \mathrm{~km} / \mathrm{h}$. The wheel speed measurements have obvious fluctuation, as shown in Figure 13, and this could cause challenges for the velocity estimation. However, the wheel speed selection can reduce the influence of these fluctuations and the estimation results of current method are satisfactory. In addition, none of the four wheels are slipping simultaneously during this test, thus the slip flag is always "off". 

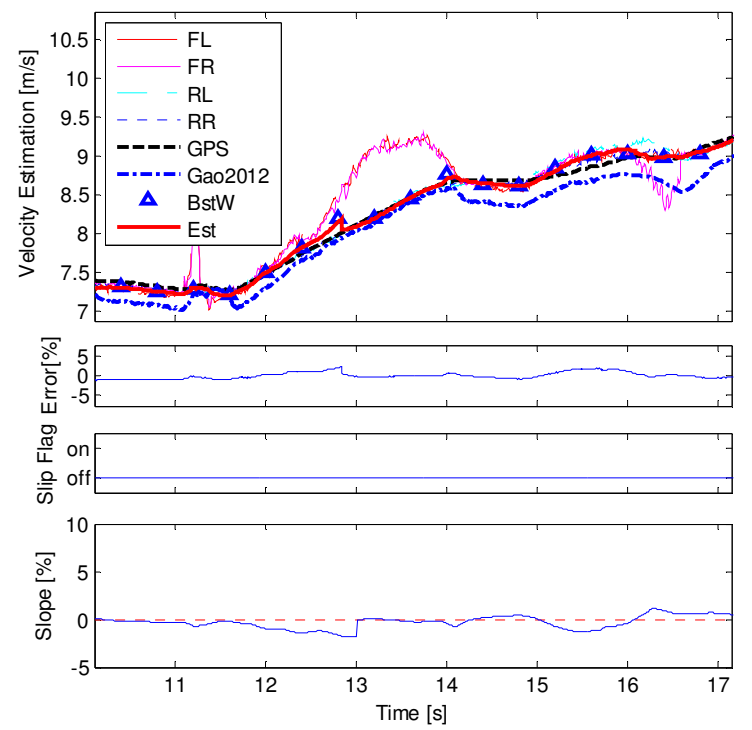

Figure 13 Test results of circle on ice
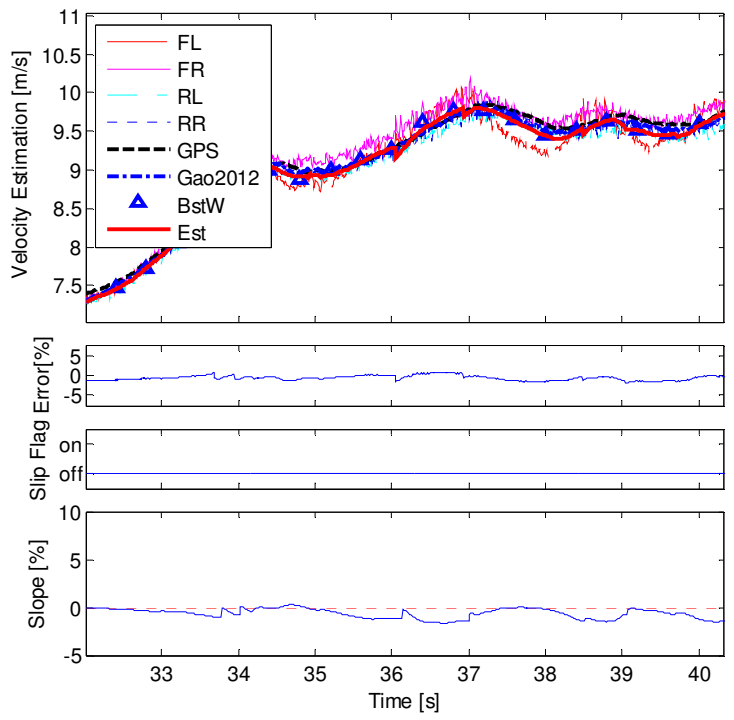

Figure 14 Test results of driving on snow

When driven on snow, the vehicle estimation is most often not too challenging since there usually is always one wheel that is close to free-rolling. In spite of there is some noise in wheel speed measurement, it can be treated as Gauss White Noise by the Kalman Filter and has no serious influence on velocity estimation. As shown in Figure 14, the current method works well in this test and also in this case the estimation error is within $\pm 5 \%$.

As shown in Figure 15, the vehicle starts on 10\% slope road from stand still. The wheels show excessive wheel slip at the beginning of this test. Even so, the wheel selection method is able to find the best wheel speed. The slip flag indicates that all the wheels are slip excessively only for a short while, and the best wheel curve is quite smooth even if some wheels are slipping. In spite of the delay of slope estimation, the velocity estimation results are as desired. When the vehicle is starting on $15 \%$ slope road, because of the low friction, it moves backward. However, the wheel speed sensor can only provide absolute measurements. In this situation, the motor speed is used to determine the direction of rotation. In Figure 16 it can be seen that the velocity receives the correct direction when the vehicle moves backward, and the estimation results are thus satisfactory.
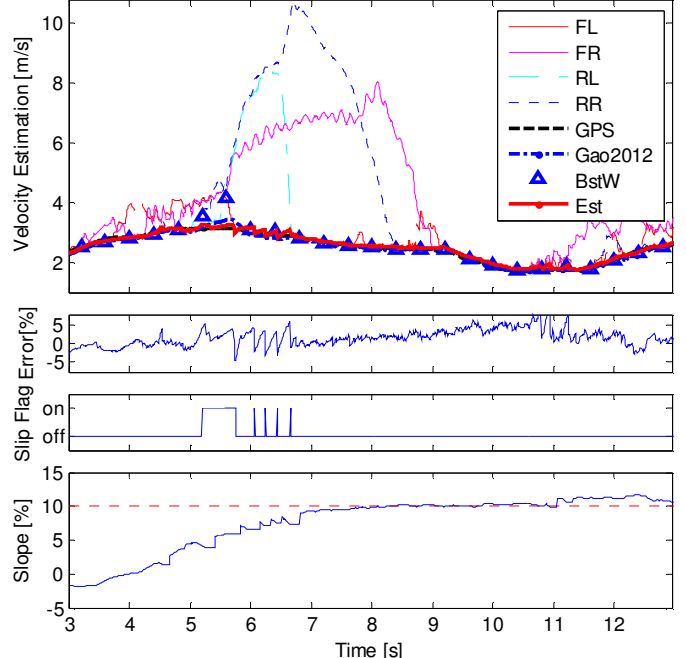

Figure 15 Test results of start on $10 \%$ slope
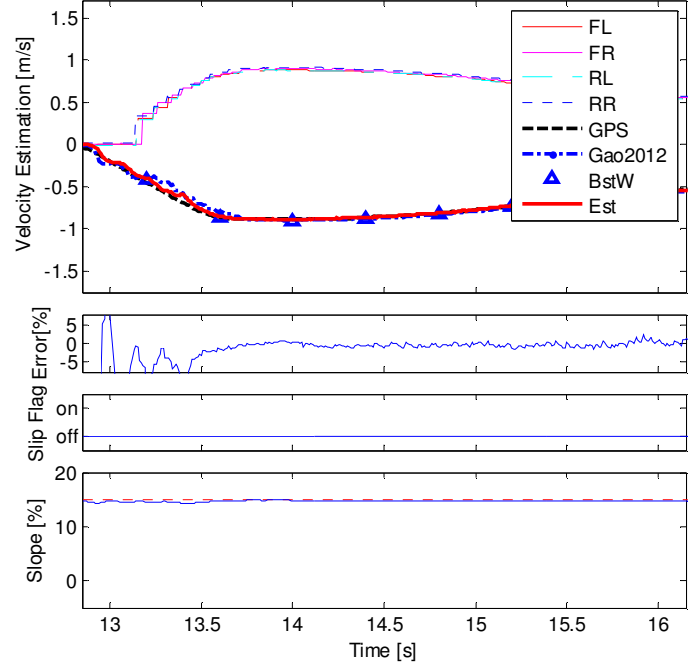

Figure 16 Test results of rolling back on 15\% slope 


\section{ACKNOWLEDGEMENTS}

The authors want to thank VINNOVA - Sweden's Innovation Agency and FKG (the Scandinavian automotive supplier association) for financially supporting this project through the FFI Vehicle Development program (grant number 2012-03682).

Furthermore, we are grateful for the support from Dr. Xiang Chu from Tongji University, Prof. Bengt Jacobson from Chalmers and e-AAM Driveline Systems in enabling this work.

\section{CONCLUSIONS}

This paper presents a method to estimate the longitudinal velocity and road slope that solves the case when wheels are slipping beyond the friction limit of the tires, so-called excessive slip. This excessive slip detection is in part achieved by introducing a novel excessive slip criteria and a wheel speed selection method.

There is an inevitable delay to detect excessive slip using the approach without wheel torque. This delay causes a large deviation between the velocity estimation and the true velocity. To solve this delay the wheel speed criterion and pre-estimation criterion is complemented by a third criterion based on the wheel torque, which can be obtained accurately from the electric motor. The wheel torque criterion can detect the slipping wheels without substantial delay to the cost of sensitivity to measurement noise. However, changes in the wheel speeds are used in addition to verify the excessive slip which implies an accurate criterion with good timing.

The velocity estimation accuracy is also affected by differences in the wheel speed measurements. In some situations, excessive slip of one wheel may lead to large estimation error and is hard to detect by the excessive slip criteria. Thus, one wheel is selected that best represent the vehicle speed and is further used as the observation variable of the Kalman filter. This approach improves the robustness of the estimate as filter is less sensitive for slipping wheels. In addition it reduces the computation in the Kalman filer as the dimension is reduced from a four dimensional problem to a scalar one.

Since this was a limited study, further development of the algorithm needs to be done to adapt to changes in wheel radius, linear-range wheel slip, variations in the friction on the different wheels and the effects of large side-slip.

In conclusion, the approach was successfully verified towards extensive winter tests under realistic conditions. The presented algorithm shows a major step forward in finding a practical method to estimate the longitudinal velocity with good performs on slippery roads even in cases when all four wheels are slipping. 


\section{REFERENCES}

[1] Xiong Lu, Gao Yunlong, Feng Yuan (Tongji University), 2012 - "Vehicle Longitudinal Velocity Estimation with Adaptive Kalman Filter" International Symposium on Advanced

[2] Song, C., Uchanski, M., and Hedrick, J., "Vehicle Speed Estimation Using Accelerometer and Wheel Speed Measurements, " SAE Technical Paper 2002-01-2229, 2002, doi:10.4271/2002-01-2229.

[3] Kobayashi, K.; Cheok, K.C.; Watanabe, K.; "Estimation of absolute vehicle speed using fuzzy logic rulebased Kalman filter," American Control Conference, Proceedings of the 1995 , vol.5, no., pp.3086-3090 vol.5, 21-23 Jun 1995, doi: 10.1109/ACC.1995.532084

[4] Daiss, A.; Kiencke, U.; , "Estimation of vehicle speed fuzzy-estimation in comparison with Kalmanfiltering," Control Applications, 1995., Proceedings of the 4th IEEE Conference on , vol., no., pp.281-284, 28-29 Sep 1995, doi: 10.1109/CCA.1995.555716

[5] Tanelli, M.; Savaresi, S.M.; Cantoni, C.; , "Longitudinal vehicle speed estimation for traction and braking control systems, " Computer Aided Control System Design, 2006 IEEE International Conference on Control Applications, 2006 IEEE International Symposium on Intelligent Control, 2006 IEEE , vol., no., pp.27902795, 4-6 Oct. 2006, doi: 10.1109/CACSD-CCA-ISIC.2006.4777080

[6] Nybacka, Mikael. "Opportunities in automotive winter testing.” In: Proceedings of the ASME International Design Engineering Technical Conferences and Computers and Information in Engineering Conference 2009 : DETC 2009. ASME Press, 2010. p. 879-886

[7] Hallnor M., Duringhof H.,-M., Klomp M., Arikere A., Hybridization and its opportunities for improved vehicle dynamics. FISITA world automobile congress 2012.

[8] Cevher, V.; Chellappa, R.; McClellan, J.H., "Vehicle Speed Estimation Using Acoustic Wave Patterns," Signal Processing, IEEE Transactions on , vol.57, no.1, pp.30,47, Jan. 2009

[9] Kruger, W.; Enkelmann, W.; Rossle, S., "Real-time estimation and tracking of optical flow vectors for obstacle detection," Intelligent Vehicles '95 Symposium., Proceedings of the , vol., no., pp.304,309, 25-26 Sep 1995

[10]Liu G "ABS system is based on data fusion technology, the speed estimation methods", Journal of Scientific Instrument, 2004.

[11] Jiang F., Gao Z. "An Adaptive Nonlinear Filter Approach to the Vehicle Velocity Estimation for ABS", Proceeding of the 2000 IEEE International Conference.

[12] Kobayashi K., Cheok K.-C., Watanabe K.“Estimation of Absolute Vehicle Speed using Fuzzy Logic RuleBased Kalman Filter". Proceedings of the American Control Conference, Seattle, Washington, June 1995:3086-3090

[13] Imsland L., Johansen T.-A., Fossen T.-I. "Nonlinear observer for vehicle velocity estimation". 2006 SAE International:2-10, 2006. 